\title{
Adaptive resource allocation and decoding strategy for underlay multi-carrier cooperative cognitive radio systems
}

\author{
Mylene Pischella* and Didier Le Ruyet \\ CNAM CEDRIC/LAETITIA, 292 rue Saint-Martin, 75003 Paris, France
}

\begin{abstract}
This paper proposes a resource allocation and decoding strategy at the secondary system for underlay and interweave multi-carrier cooperative cognitive radio. The objective is to maximise the sum rate of both primary and secondary systems, taking into account the interference threshold constraint and the fact that the primary receiver always considers interference as noise. The decoding method at the secondary receivers is either Successive Interference Cancellation or treating interference as noise, depending on their channel gains. The rationale for this strategy is the extension of the Han-Kobayashi capacity-achieving strategy on the Gaussian interference channel to the studied cognitive scenario. The proposed resource allocation algorithm is composed of a subcarrier allocation procedure to avoid intercell interference between secondary cells and of an iterative power allocation algorithm that maximises the sum rate on the primary and secondary cells, respectively. The proposed algorithm achieves high data rate improvements for the secondary systems while maintaining a low degradation on the primary system's rate. It also benefits from multi-user diversity and is robust to the distance variations. Copyright (C) 2013 John Wiley \& Sons, Ltd.
\end{abstract}

\author{
*Correspondence \\ Mylene Pischella, CNAM CEDRIC/LAETITIA, 292 rue Saint-Martin, 75003 Paris, France. \\ E-mail: mylene.pischella@cnam.fr
}

Received 24 January 2013; Revised 28 May 2013; Accepted 20 September 2013

\section{INTRODUCTION}

The need for cognitive radio has emerged in the last decade because new wireless systems are currently deployed, and vacant spectrum is becoming scarce $[1,2]$. The spectrum utilisation is not efficient in most licensed systems, with high variations depending on time, frequency and location. Cognitive radio systems are defined as wireless communication systems that intelligently utilize any available side information of the other nodes with which they share the spectrum [3]. The cognitive system is consequently aware of its environment [4]. Three types of cognitive radio systems exist: interweave, underlay and overlay cognitive radio. Interweave and underlay systems are typically deployed in a bandwidth, which is occupied by a licensed system, called the primary system. The cognitive system is then referred to as the secondary system. It can access to the spectrum only if it does not degrade the primary system's rate: if no degradation is allowed, then the secondary system only uses interweave and transmits in the subbands that are currently left vacant by the primary system. If some degradation on the primary system's rate is allowed, then the secondary system may transmit in the same subbands but the interference that it generates to the primary system should be below a given threshold. Finally, overlay cognitive radio assumes that the secondary system knows the primary system's codebook, channel gains and its transmitted messages. From the point-of-view of information theory, it is an interference channel with asymmetric channel knowledge [5,6]. Several encoding techniques have been obtained for different cases [7], such as rate splitting, dirty paper coding and partial relaying by the secondary system [3].

The capacity of the secondary system with underlay cognitive radio has been studied in [8-10] for Additive White Gaussian Noise (AWGN) or fading channels with one transmitter or for Gaussian multiple access channels (MAC). The interference threshold at the primary receiver is translated as a transmit power constraint at the secondary transmitter or at the set of secondary transmitters for MAC. For the single-secondary transmitter case, Gaussian codebooks are optimal and the primary transmitter's signal should be treated as noise at the secondary receiver. The same result applies to the MAC case, considering a sum transmit power constraint on all secondary transmitters. These results however do not take into account the 
global capacity of the system. In this paper, we are interested in maximising the sum of the data rates of the primary system and of the secondary system. Adding this objective of sum-rate maximisation complexifies the initial problem, which then becomes a Gaussian interference channel (G-IFC) capacity determination problem, with additional constraints. Our objective here is not to solve it with information-theoretic tools but rather to propose a good heuristic that is suitable for multi-carrier systems.

The studied scenario is the following: we consider a multi-carrier interweave and underlay cognitive radio system where a secondary system composed of one or two transmitter-receiver links tries to access the primary system's band under an interference threshold limitation. The primary system remains unaware of the secondary's presence and thus always treats interference as noise. Each secondary receiver adapts its decoding strategy, depending on whether its received interference is weak or strong. If interference is weak, the secondary receiver treats it as noise; whereas if it is strong, the secondary receiver uses Successive Interference Cancellation (SIC), first decoding the primary user's message and then its own. The rationale for determining which decoding strategy should be used is the information-theoretic results from Han and Kobayashi on the two-user G-IFC [11]. The Han-Kobayashi strategy involves splitting each link information into two parts: the common information, which will be decoded by both receivers, and the private information, which is only decoded by the receiver of interest. It is optimal in almost all cases $[12,13]$. An iterative power allocation and decoding strategy has been proposed for the multi-carrier two-user G-IFC in [14], providing additional insight into the multi-carrier G-IFC with respect to [15]. These results on the two-user G-IFC do not directly extend to the studied cognitive case. In the absence of known results on the achievable rate region of the multi-carrier underlay cognitive system considering also the primary rate, we will extend the G-IFC decoding strategy to this specific case, taking into account its characteristics.

When several secondary transmitter-receiver links are present, they cooperate in order to transmit in different subcarriers so that they do not interfere each other. The proposed subcarrier allocation algorithm makes use of the decoding regions and estimates the achievable data rate in each subcarrier. Power allocation is then optimised depending on this strategy. It is shown that, by decomposing the initial problem into a set of power allocation problems at the primary and secondary systems, respectively, and by using an iterative process where the power of each transmitter is updated depending on the power values set at the previous iteration, the local optimization problem at each transmitter becomes convex. It can then be easily solved by water-filling.

The algorithm presented in this paper is quite different from the usual resource allocation heuristics proposed in the literature, where interference is always treated as noise [16-19] and where the data rate of the primary system is not maximised but only protected by the interference threshold.

The novelty of the paper lies in the establishment of a link between information theory and resource allocation for cognitive radio. Most papers in resource allocation consider interference as noise, whereas most informationtheoretical studies assume fixed power values. In this paper, both issues are jointly considered, which provide some additional gain. In details, the main contributions of the paper are as follows:

- For the underlay and interweave cognitive radio system, a heuristic is determined in order to propose a practical decoding strategy for the secondary receivers. It is based on the study of the achievable rate regions of the two-user G-IFC and on the requirements of the cognitive system in terms of channel knowledge and interference threshold at the primary receiver.

- A resource allocation algorithm composed of subcarrier allocation and power allocation is then proposed. It is first derived with one secondary cell and then with two secondary cells. In that case, subcarrier allocation requires cooperation between the secondary systems. Power allocation is iteratively performed independently on each cell.

- Thorough numerical results are provided to assess the performances of the proposed algorithm, with one or two secondary cells and with varying locations of the secondary base stations (BSs). They show the relevance of this algorithm compared with several standard algorithms.

The paper is organized as follows. Section 2 describes the system model and provides a summary of the achievable rate regions on the two-user interference channel. Section 3 details the proposed power allocation and decoding strategy for underlay cognitive systems with one secondary cell. Section 4 then extends this strategy to a different scenario, with two cooperating secondary cells, where only one secondary cell is active in each subcarrier, and the cells cooperate for subcarrier allocation. Section 5 evaluates the performances of the proposed algorithm and compares them with several other methods, in the two considered scenario. Finally, Section 6 concludes the paper.

\section{SYSTEM MODEL AND DECODING STRATEGIES}

\subsection{System model}

We consider a multi-carrier cognitive network composed of a primary cell and one or two secondary cells. The secondary cells use channel underlay and channel interweave: they either transmit in subcarriers under a given interference threshold for the primary system or transmit in the subcarriers where the primary system is not active. We study the uplink transmission in a given time slot. Only one 


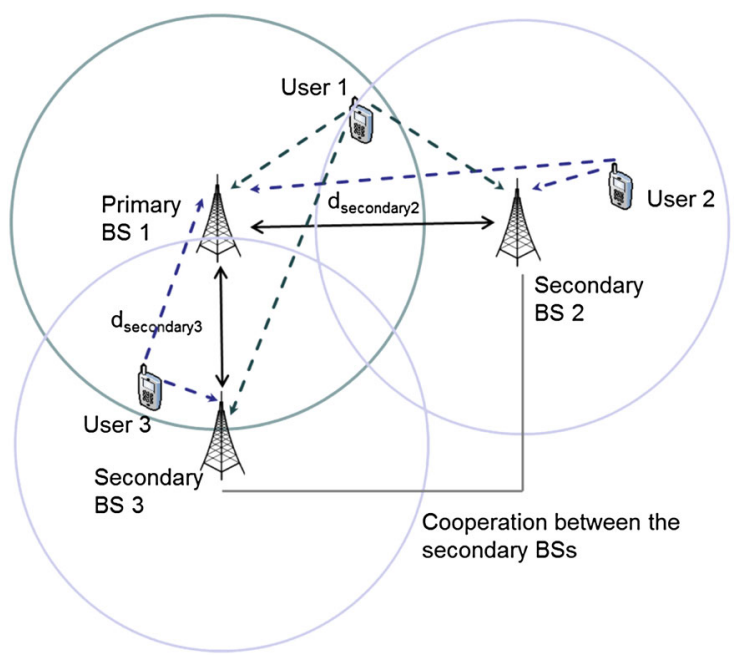

Figure 1. Considered scenario with two secondary cells.

user is then active in each cell. Index 1 refers to the primary system, and indexes 2 and 3 refer to the secondary systems. The primary system occupies a licensed bandwidth $B$ with $L$ adjacent subcarriers in an Orthogonal Frequency Division Multiplex system with perfect synchronisation. The secondary BSs are located at distances $d_{\text {secondary, } 2}$ and $d_{\text {secondary, } 3}$ from the primary BS, respectively, and try to access the same bandwidth $B$. The secondary BS locations are orthogonal to each other with respect to the primary BS location (Figure 1). In the following, we will study two scenarios. In the first one, only one secondary BS is present (BS 2). In the second one, the two secondary cells are cooperating for multiple access: in each subcarrier, only the secondary user with the highest estimated achievable data rate is allowed to transmit. Consequently, the two secondary cells do not interfere each other. This section first details both scenarios.

\subsubsection{System model with one secondary cell.}

In each subcarrier $k \in\{1, . ., L\}$, if the power per subcarrier is known, the channel is a two-user G-IFC with additional constraints due to the cognitive scenario (Figure 2):

$$
\begin{aligned}
& Y_{1}^{k}=h_{11}^{k} X_{1}^{k}+h_{12}^{k} X_{2}^{k}+Z_{1}^{k} \\
& Y_{2}^{k}=h_{21}^{k} X_{1}^{k}+h_{22}^{k} X_{2}^{k}+Z_{2}^{k}
\end{aligned}
$$

where $Y_{i}^{k}$ is the channel output and $X_{i}^{k}$ is the channel input corresponding to data $\mathbf{s}_{i}^{k}$ with power $P_{i}^{k} . h_{i j}^{k}$ is the channel gain between transmitter $j$ and receiver $i$, with $(i, j) \in\{1,2\}^{2}$. The noise $Z_{i}^{k}$ is independent and identically distributed Gaussian with variance $n_{i}^{k}=n_{0}$, assumed equal on each subcarrier and at each receiver. We denote by $R_{i}^{k}$ the data rate on link $i$ in subcarrier $k . \mathbf{P}_{i}=\left[P_{i}^{1}, P_{i}^{2}, \ldots, P_{i}^{L}\right]^{\prime}$ is the vector of powers for transmitter $i$.

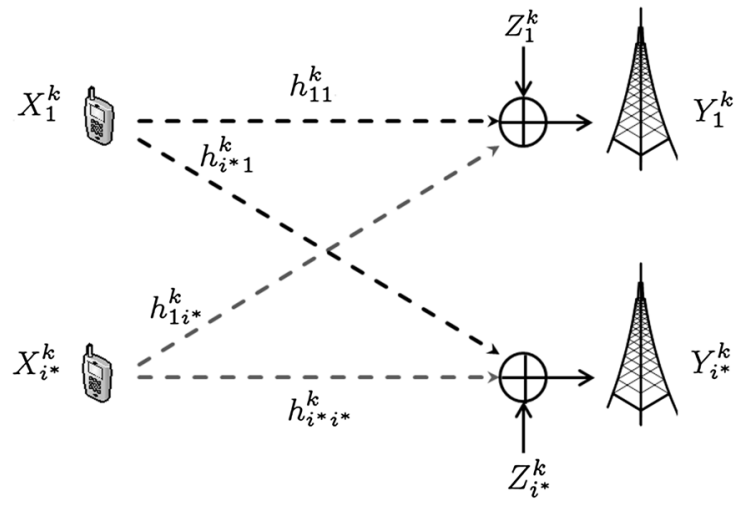

Figure 2. Two-user Gaussian interference channel on subcarrier

$$
k \in\{1, . ., L\} \text {. }
$$

Each transmitter has a maximum sum power constraint on all its subcarriers, denoted by $P_{i, \max }$ for user $i$. Thus, the overall channel is not a series of parallel G-IFC, because the sum power must be spread over all subcarriers.

Finally, the secondary user cannot transmit at a higher power level than $I_{\mathrm{th}}^{k} /\left|h_{12}^{k}\right|^{2}$, where $I_{\mathrm{th}}^{k}$ is the maximum interference level allowed on the primary user. We will assume perfect channel state information (CSI) at the primary and secondary transmitters and receivers on their respective links. The secondary system also needs to know the primary's direct and interfering channel gains and its modulation and coding scheme in order to perform adaptive decoding and power allocation. Channel and power knowledge can be implemented in practice by exploiting channel reciprocity, or by partial cooperation of the primary system, that would send its direct channel gain and its modulation and coding scheme to the secondary system.

\subsubsection{System model with two cooperating secondary cells.}

In the second scenario, the secondary cells 2 and 3 are cooperating for multiple access. On the basis of the estimated data rate that can be achieved by each user 2 and 3 in each subcarrier $k \in\{1, \ldots, L\}$, only the secondary user with the highest $\tilde{R}_{i}^{k}$ is allowed to transmit. The details of how rates are estimated will be provided in Section 4.2. The channel in each subcarrier is consequently still a two-user G-IFC :

$$
\begin{aligned}
Y_{1}^{k} & =h_{11}^{k} X_{1}^{k}+h_{1 i^{*}}^{k} X_{i^{*}}^{k}+Z_{1}^{k} \\
Y_{i^{*}}^{k} & =h_{i^{*} 1}^{k} X_{1}^{k}+h_{i^{*} i^{*}}^{k} X_{i^{*}}^{k}+Z_{i^{*}}^{k} \\
i^{*} & =\arg \max _{i \in\{2,3\}} \tilde{R}_{i}^{k}
\end{aligned}
$$

The same notations as previously stand, with $i \in\{2,3\}$. The maximum interference constraint becomes $P_{i}^{k} \leqslant$ $I_{\mathrm{th}}^{k} /\left|h_{1 i *}^{k}\right|^{2}$. On top of the CSI requirements of the first 
scenario that are still valid, we also assume that both secondary cells exchange their evaluated rates $\tilde{R}_{i}^{k}$ in each subcarrier. This can be performed if both BSs that are connected by a backhaul or through a specific signalling protocol between the secondary users if there is none.

\subsection{Review of the achievable rate region on the two-user Gaussian interference channel}

The proposed decoding strategy in the cognitive scenarios is based on the rate regions that can be achieved using Han-Kobayashi strategy, and that, in some cases, are sumcapacity achieving for the two-user G-IFC. Consequently, in this section, we review these strategies and the corresponding rates. To simplify notations, we consider the two-user G-IFC (1) on subcarrier $k$ and assume that the powers are set to fixed values. On this channel, both links 1 and 2 are treated equally, and there is no cognitive interference constraint. Several cases must be distinguished: weak, strong and mixed interference.

\subsubsection{Weak interference.}

Interference is weak if $\left|h_{12}^{k}\right|^{2} \leqslant\left|h_{11}^{k}\right|^{2}$ and $\left|h_{21}^{k}\right|^{2} \leqslant$ $\left|h_{22}^{k}\right|^{2}$. The capacity region is not known for the general weak interference case. It has however been shown in $[12,13]$ that if interference is very weak, the sum capacity is reached when treating interference as noise at both receivers, that is, sending only private information. The very weak interference condition is

$$
\begin{aligned}
& \sqrt{\frac{\left|h_{12}^{k}\right|^{2}}{\left|h_{11}^{k}\right|^{2}}\left(\frac{\left|h_{21}^{k}\right|^{2}}{\left|h_{22}^{k}\right|^{2}} \frac{\left|h_{11}^{k}\right|^{2}}{n_{0}} P_{1}^{k}+1\right)} \\
& +\sqrt{\frac{\left|h_{21}^{k}\right|^{2}}{\left|h_{22}^{k}\right|^{2}}}\left(\frac{\left|h_{12}^{k}\right|^{2}}{\left|h_{11}^{k}\right|^{2}} \frac{\left|h_{22}^{k}\right|^{2}}{n_{0}} P_{2}^{k}+1\right) \leqslant 1
\end{aligned}
$$

The individual data rates are then

$$
\begin{aligned}
& R_{1}^{k}=\frac{B}{L} \log _{2}\left(1+\frac{\left|h_{11}^{k}\right|^{2} P_{1}^{k}}{n_{0}+\left|h_{12}^{k}\right|^{2} P_{2}^{k}}\right) \\
& R_{2}^{k}=\frac{B}{L} \log _{2}\left(1+\frac{\left|h_{22}^{k}\right|^{2} P_{2}^{k}}{n_{0}+\left|h_{21}^{k}\right|^{2} P_{1}^{k}}\right)
\end{aligned}
$$

If interference is weak, but not very weak, this strategy is no longer optimal $[20,21]$, and how to achieve the sum capacity is still an open issue.

\subsubsection{Strong interference.}

The G-IFC is in strong interference conditions when $\left|h_{12}^{k}\right|^{2} \geqslant\left|h_{11}^{k}\right|^{2}$ and $\left|h_{21}^{k}\right|^{2} \geqslant\left|h_{22}^{k}\right|^{2}$. Then, the optimal capacity region is achieved by sending common information only $[11,22]$.

Under very strong interference conditions $\frac{\left|h_{12}^{k}\right|^{2}}{\left|h_{11}^{k}\right|^{2}} \geqslant 1+$ $\frac{\left|h_{11}^{k}\right|^{2}}{n_{0}}$ and $\frac{\left|h_{21}^{k}\right|^{2}}{\left|h_{22}^{k}\right|^{2}} \geqslant 1+\frac{\left|h_{22}^{k}\right|^{2}}{n_{0}}$, all interference can be removed by using SIC at both receivers. The individual capacities are consequently interference-free.

Under strong but not very strong conditions, the sumcapacity is equal to the minimum of the two compound MACs formed at each receiver:

$$
\begin{aligned}
& R_{1}^{k} \leqslant \frac{B}{L} \log _{2}\left(1+\frac{\left|h_{11}^{k}\right|^{2}}{n_{0}} P_{1}^{k}\right) \\
& R_{2}^{k} \leqslant \frac{B}{L} \log _{2}\left(1+\frac{\left|h_{22}^{k}\right|^{2}}{n_{0}} P_{2}^{k}\right) \\
& R_{1}^{k}+R_{2}^{k} \leqslant \frac{B}{L} \min \left\{\log _{2}\left(1+\frac{\left|h_{11}^{k}\right|^{2}}{n_{0}} P_{1}^{k}+\frac{\left|h_{12}^{k}\right|^{2}}{n_{0}} P_{2}^{k}\right) ;\right. \\
&\left.\log _{2}\left(1+\frac{\left|h_{21}^{k}\right|^{2}}{n_{0}} P_{1}^{k}+\frac{\left|h_{22}^{k}\right|^{2}}{n_{0}} P_{2}^{k}\right)\right\}
\end{aligned}
$$

If $\left|h_{11}^{k}\right|^{2} P_{1}^{k}+\left|h_{12}^{k}\right|^{2} P_{2}^{k} \geqslant\left|h_{21}^{k}\right|^{2} P_{1}^{k}+\left|h_{22}^{k}\right|^{2} P_{2}^{k}$, the sum-capacity is obtained by using SIC at receiver 2 , where $\mathbf{s}_{1}^{k}$ is decoded first, considering $\mathbf{s}_{2}^{k}$ as noise, and then removed from the total signal, which allows $\mathbf{s}_{2}^{k}$ to be decoded interference-free. This operating point, also chosen for MAC rates at receiver 1, corresponds to the following individual capacities:

$$
\begin{aligned}
& R_{1}^{k}=\frac{B}{L} \log _{2}\left(1+\frac{\left|h_{21}^{k}\right|^{2} P_{1}^{k}}{n_{0}+\left|h_{22}^{k}\right|^{2} P_{2}^{k}}\right) \\
& R_{2}^{k}=\frac{B}{L} \log _{2}\left(1+\frac{\left|h_{22}^{k}\right|^{2}}{n_{0}} P_{2}^{k}\right)
\end{aligned}
$$

It should be noted that this is not the only pair of rates leading to the sum capacity but the easiest one because it does not require time-sharing at receiver 2 .

If $\left|h_{11}^{k}\right|^{2} P_{1}^{k}+\left|h_{12}^{k}\right|^{2} P_{2}^{k} \leqslant\left|h_{21}^{k}\right|^{2} P_{1}^{k}+\left|h_{22}^{k}\right|^{2} P_{2}^{k}$, the sum-capacity is obtained by using SIC in the opposite way, decoding $\mathbf{s}_{2}^{k}$ prior to $\mathbf{s}_{1}^{k}$ at receiver 1 and setting the same rates at receiver 2 . The operating point is deduced by inverting indexes in Equation (6). 


\subsubsection{Mixed interference.}

The G-IFC is mixed when the interfering gain is higher than the direct gain at one receiver, and the interfering gain is lower than the direct gain at the other receiver. In that case, it has been shown in [13] that the sum-capacity is achieved by transmitting only common information for the highly interfering link and only private information for the other one.

If $\left|h_{12}^{k}\right|^{2} \leqslant\left|h_{11}^{k}\right|^{2}$ and $\left|h_{21}^{k}\right|^{2} \geqslant\left|h_{22}^{k}\right|^{2}$, the sum capacity is achieved if $\mathbf{s}_{1}^{k}$ is common information, and $\mathbf{s}_{2}^{k}$ is private information. Consequently, receiver 2 decodes both messages and receiver 1 only decodes $\mathbf{s}_{1}^{k}$.

The rate constraints are a compound of the MAC constraints at receiver $2,(C 2)-(C 4)$, and of the constraint at receiver $1,(C 1)$, which is because receiver 1 considers interference as noise:

$$
\begin{aligned}
& R_{1}^{k} \leqslant \frac{B}{L} \log _{2}\left(1+\frac{\left|h_{11}^{k}\right|^{2} P_{1}^{k}}{n_{0}+\left|h_{12}^{k}\right|^{2} P_{2}^{k}}\right)(C 1) \\
& R_{1}^{k} \leqslant \frac{B}{L} \log _{2}\left(1+\frac{\left|h_{21}^{k}\right|^{2} P_{1}^{k}}{n_{0}}\right)(C 2) \\
& R_{2}^{k} \leqslant \frac{B}{L} \log _{2}\left(1+\frac{\left|h_{22}^{k}\right|^{2} P_{2}^{k}}{n_{0}}\right)(C 3) \\
& R_{1}^{k}+R_{2}^{k} \leqslant \frac{B}{L} \log _{2}\left(1+\frac{\left|h_{21}^{k}\right|^{2} P_{1}^{k}+\left|h_{22}^{k}\right|^{2} P_{2}^{k}}{n_{0}}\right)
\end{aligned}
$$

An operating point reaching the sum capacity is

$$
\begin{aligned}
& R_{1}^{k}=\frac{B}{L} \min \left\{\log _{2}\left(1+\frac{\left|h_{21}^{k}\right|^{2} P_{1}^{k}}{n_{0}+\left|h_{22}^{k}\right|^{2} P_{2}^{k}}\right) ;\right. \\
& \left.\log _{2}\left(1+\frac{\left|h_{11}^{k}\right|^{2} P_{1}^{k}}{n_{0}+\left|h_{12}^{k}\right|^{2} P_{2}^{k}}\right)\right\} \\
& R_{2}^{k}=\frac{B}{L} \log _{2}\left(1+\frac{\left|h_{22}^{k}\right|^{2}}{n_{0}} P_{2}^{k}\right)
\end{aligned}
$$

Similarly, if $\left|h_{12}^{k}\right|^{2} \geqslant\left|h_{11}^{k}\right|^{2}$ and $\left|h_{21}^{k}\right|^{2} \leqslant\left|h_{22}^{k}\right|^{2}$, the sum capacity is achieved if $\mathbf{s}_{2}^{k}$ is common information, and $\mathbf{s}_{1}^{k}$ is private information. The data rates are obtained by symmetry with respect to Equation (8).

\subsection{Introduction of the cognitive constraints in the two-user Gaussian interference channel}

In the studied cognitive scenario, the primary receiver always considers interference as noise and thus cannot adapt its decoding strategy. $\mathbf{s}_{2}^{k}$ is consequently always private information. The Han-Kobayashi strategies on the two-user G-IFC achieve the sum-capacity by using private information for $\mathbf{s}_{2}^{k}$ in the following cases: when interference is very weak (Equation (3)), and with mixed interference; when interference is strong at receiver 2 but weak at receiver 1 . These two cases are however rare (around $7 \%$ of the cases with Rayleigh fadings for the two-user GIFC according to [14]) and restricting to them would lead to very low data rates for the secondary system. Consequently, we propose to adapt the decoding strategy in the following way for the two-user cognitive system:

- When $P_{1}^{k}=0$, the secondary system uses channel interweave. It transmits in subcarrier $k$ with no primary interference threshold.

- When $P_{1}^{k} \neq 0$ and interference is weak at the secondary receiver: $\left|h_{21}^{k}\right|^{2} \leqslant\left|h_{22}^{k}\right|^{2}$, the secondary receiver treats it as noise. Its maximum transmit power is limited by the primary interference threshold.

- When $P_{1}^{k} \neq 0$ and interference is strong at the secondary receiver: $\left|h_{21}^{k}\right|^{2}>\left|h_{22}^{k}\right|^{2}$, the secondary receiver uses SIC, decoding first $\mathbf{s}_{1}^{k}$ and then $\mathbf{s}_{2}^{k}$. Its maximum transmit power is limited by the primary interference threshold.

The proposed strategy is a heuristic and will not lead to a maximisation of the sum rate in the general cases. However, the simulation results in Sections 4 and 5 show that it can achieve a good trade-off in most situations, providing high-enough data rates for the secondary systems at the expense of very low degradation of the primary system's rates.

\section{POWER ALLOCATION AND DECODING STRATEGY WITH ONE SECONDARY CELL}

\subsection{Studied scenario and description of the strategy}

In this section, we propose a power allocation algorithm that makes use of the decoding strategy proposed in Section 2.3, for the scenario detailed in Section 2.1.1 and the system model given by Equation (1) with one secondary cell.

Two optimization objectives are considered, at the primary system and at the secondary system. First, the objective is to maximise the sum rate in the primary cell. 
Then, the second objective is to maximise the sum rate in the secondary cell, given that the interference generated by the secondary transmitter on the primary receiver should not exceed a threshold $I_{\text {th }}^{k}$ per subcarrier, except in the subcarriers where the primary receiver is not active.

An iterative algorithm is used. At each iteration $n$, the primary transmitter first performs power optimization (based on the interference at the primary receiver in the previous iteration, $n-1$ ), and then, the secondary link adapts its decoding strategy and performs power optimization based on the interference received at $n-1$. A series of optimization problems is consequently obtained. Each of them will be shown to be convex in the next subsections.

\subsection{Power allocation problem for the primary user}

At iteration $n$, the primary user must solve the following power allocation problem:

$$
\begin{aligned}
& \max _{\left\{\mathbf{P}_{1, n}\right\}} \frac{B}{L} \sum_{k=1}^{L} \log _{2}\left(1+b_{1,(n-1)}^{k} P_{1, n}^{k}\right) \\
& \text { s.t. } \sum_{k=1}^{L} P_{1}^{k} \leqslant P_{1, \max } \\
& \text { s.t. } P_{1}^{k} \geqslant 0 \forall k \in\{1, \ldots, L\}
\end{aligned}
$$

with $b_{1,(n-1)}^{k}=\left|h_{11}^{k}\right|^{2} /\left(n_{0}+\left|h_{12}^{k}\right|^{2} P_{2,(n-1)}^{k}\right)$.

This optimization problem is convex and its solution is given by

$$
P_{1, n}^{k}= \begin{cases}0 & \text { if } b_{1,(n-1)}^{k}=0 \\ {\left[\frac{1}{\mu_{1, n}}-\frac{1}{b_{1,(n-1)}^{k}}\right]^{+}} & \text {otherwise }\end{cases}
$$

where $\mu_{1, n}$ is a constant set in order to fulfil the sum power constraint, $\sum_{k=1}^{L} P_{1}^{k}=P_{1, \max }$, and $[x]^{+}=\max \{x, 0\}$.

As said in Section 2.1.1, the primary transmitter has full channel knowledge; it also evaluates its received noise plus interference at each iteration to obtain $b_{1,(n-1)}^{k}$. This estimation is assumed perfect.

\subsection{Decoding strategy for the secondary user}

Before setting the optimization problem, the achievable rates depending on the cases are first determined.

- Channel interweave: when $P_{1}^{k}=0$, the secondary receiver transmits with no interference threshold limitation, and its rate is interference-free.

- Weak interference at the secondary receiver: When interference is weak at the secondary receiver, $\left|h_{21}^{k}\right|^{2} \leqslant\left|h_{22}^{k}\right|^{2}$ (and $P_{1}^{k} \neq 0$ ), it is treated as noise at the secondary receiver. The data rate is then

$$
R_{2}^{k}=\frac{B}{L} \log _{2}\left(1+\frac{\left|h_{22}^{k}\right|^{2} P_{2}^{k}}{n_{0}+\left|h_{21}^{k}\right|^{2} P_{1}^{k}}\right)
$$

- Strong interference at the secondary receiver: When $\left|h_{21}^{k}\right|^{2}>\left|h_{22}^{k}\right|^{2}$ (and $P_{1}^{k} \neq 0$ ), the secondary receiver decodes both messages. The rate constraints are given by Equation (7). Then, because $\mathbf{s}_{1}^{k}$ is decoded first and time-sharing is not authorised, constraints $(C 2)$ and $(C 4)$ of Equation (7) can be merged into a new constraint $\left(C 2^{\prime}\right)$, leading to the following set of constraints:

$$
\begin{aligned}
& R_{1}^{k} \leqslant \frac{B}{L} \log _{2}\left(1+\frac{\left|h_{11}^{k}\right|^{2} P_{1}^{k}}{n_{0}+\left|h_{12}^{k}\right|^{2} P_{2}^{k}}\right) \\
& R_{1}^{k} \leqslant \frac{B}{L} \log _{2}\left(1+\frac{\left|h_{21}^{k}\right|^{2} P_{1}^{k}}{n_{0}+\left|h_{22}^{k}\right|^{2} P_{2}^{k}}\right) \\
& R_{2}^{k} \leqslant \frac{B}{L} \log _{2}\left(1+\frac{\left|h_{22}^{k}\right|^{2} P_{2}^{k}}{n_{0}}\right)
\end{aligned}
$$

The primary system always treats interference as noise. Consequently, the achieved data rate for the primary link is exactly given by $(C 1)$. Constraint $\left(C 2^{\prime}\right)$ is then only feasible if

$$
\frac{\left|h_{11}^{k}\right|^{2} P_{1}^{k}}{n_{0}+\left|h_{12}^{k}\right|^{2} P_{2}^{k}} \leqslant \frac{\left|h_{21}^{k}\right|^{2} P_{1}^{k}}{n_{0}+\left|h_{22}^{k}\right|^{2} P_{2}^{k}}
$$

Condition (11) can be equivalently written as

$$
\begin{aligned}
& P_{2}^{k}\left(\left|h_{21}^{k}\right|^{2}\left|h_{12}^{k}\right|^{2}-\left|h_{11}^{k}\right|^{2}\left|h_{22}^{k}\right|^{2}\right) \\
& \geqslant n_{0}\left(\left|h_{11}^{k}\right|^{2}-\left|h_{21}^{k}\right|^{2}\right)
\end{aligned}
$$

Under constraint (12), $\mathbf{s}_{1}^{k}$ is decoded error-free at the secondary receiver, and $R_{2}^{k}=B / L \log _{2}$ $\left(1+\left|h_{22}^{k}\right|^{2} P_{2}^{k} / n_{0}\right)$ can be achieved after removing $\mathbf{s}_{1}^{k}$ from the received signal.

\subsection{Power allocation problem for the secondary user}

At iteration $n$, the power allocation problem for the secondary user depends on the transmission and decoding 
Table I. Optimization coefficients for problem (13).

\begin{tabular}{lc}
\hline Cases & $b_{2,(n-1)}^{k}$ \\
$\left|h_{21}^{k}\right|^{2} \leqslant\left|h_{22}^{k}\right|^{2}$ & $\frac{\left|h_{22}^{k}\right|^{2}}{n_{0}+\left|h_{21}^{k}\right|^{2} P_{1,(n-1)}^{k}}$ \\
and $P_{1,(n-1)}^{k} \neq 0$ & $\frac{\left|h_{22}^{k}\right|^{2}}{n_{0}}$ \\
$\left|h_{21}^{k}\right|^{2}>\left|h_{22}^{k}\right|^{2}$ & $\frac{\left|h_{22}^{k}\right|^{2}}{n_{0}}$ \\
and $P_{1,(n-1)}^{k} \neq 0$ & $a_{2}^{k}$ \\
$P_{1,(n-1)}^{k}=0$ & $\left|h_{21}^{k}\right|^{2}\left|h_{12}^{k}\right|^{2}-\left|h_{11}^{k}\right|^{2}\left|h_{22}^{k}\right|^{2}$ \\
\hline Cases & 0 \\
$\left|h_{21}^{k}\right|^{2} \geqslant\left|h_{22}^{k}\right|^{2}$ & $c_{2}^{k}$ \\
and $P_{1,(n-1)}^{k} \neq 0$ & $n_{0}\left(\left|h_{11}^{k}\right|^{2}-\left|h_{21}^{k}\right|^{2}\right)$ \\
all other cases & 0 \\
\hline Cases & \\
$\left|h_{21}^{k}\right|^{2} \geqslant\left|h_{22}^{k}\right|^{2}$ & \\
and $P_{1,(n-1)}^{k} \neq 0$ & all other cases
\end{tabular}

strategy chosen on each subcarrier and can be simply written as

$$
\begin{aligned}
& \max _{\left\{\mathbf{P}_{2, n}\right\}} \frac{B}{L} \sum_{k=1}^{L} \log _{2}\left(1+b_{2,(n-1)}^{k} P_{2, n}^{k}\right) \\
& \text { s.t. } a_{2}^{k} P_{2}^{k} \geqslant c_{2}^{k} \forall k \in\{1, \ldots, L\} \\
& \text { s.t. }\left|h_{12}^{k}\right|^{2} P_{2}^{k} \leqslant I_{\text {th }}^{k} \forall k \in\{1, \ldots, L\} \text { if } P_{1,(n-1)}^{k}>0 \\
& \text { s.t. } P_{2}^{k} \geqslant 0 \forall k \in\{1, \ldots, L\} \\
& \text { s.t. } \sum_{k=1}^{L} P_{2}^{k} \leqslant P_{2, \max }
\end{aligned}
$$

Where the first constraint corresponds to condition (12), and the second one is the interference limitation constraint. The values of $b_{2,(n-1)}^{k}, a_{2}^{k}$ and $c_{2}^{k}$ depend on the decoding strategy and are given in Table I.

As said in Section 2.1.1, the secondary transmitter has full channel knowledge on both primary and receiver channels. The received interference is evaluated at each iteration, and this process is assumed perfect.

The interference limitation threshold is set according to the rate degradation that the primary user can accept [23] with respect to its maximum rate $R_{1, \max }^{k}=$ $B / L \log _{2}\left(1+\left|h_{11}^{k}\right|^{2} P_{1, n}^{k} / n_{0}\right)$. The allowed degradation, $\lambda$, is defined as

$$
(1-\lambda) R_{1, \max }^{k}=\frac{B}{L} \log _{2}\left(1+\frac{\left|h_{11}^{k}\right|^{2} P_{1, n}^{k}}{n_{0}+I_{\mathrm{th}}^{k}}\right)
$$

and $I_{\mathrm{th}}^{k}$ is obtained by inverting Equation (14).

Problem (13) is convex in $\mathbf{P}_{2, n}$. An analytical solution can be obtained with the Karush-Kuhn-Tucker conditions [24]. The solution is given in Table II. Six cases can be distinguished: in interweave (VW) case, the solution is directly provided by waterfilling, whereas the additional interference constraint limits the waterfilling solution in the weak interference (Weak Int) case. When interference is strong at the secondary receiver, four cases coexist: in SIC 1 case, constraint (12) is not feasible, which means that SIC cannot be performed because the primary rate at the secondary receiver is too low. The secondary user thus does not transmit in this subcarrier. In SIC 3 case, $\left\{a_{2}^{k}>0 ; c_{2}^{k}<0\right\}$, condition (12) is always true, and the solution is the same as in the weak interference case. In SIC 2 case, because $\left\{a_{2}^{k}<0 ; c_{2}^{k}<0\right\}$, condition (12) becomes $P_{2}^{k} \leqslant c_{2}^{k} / a_{2}^{k}$, which implies that the waterfilling solution is upper bounded by the minimum between the interference constraint and $c_{2}^{k} / a_{2}^{k}$. Finally, in SIC 4 case,

\begin{tabular}{|c|c|}
\hline Conditions & $P_{2, n}^{k}$ \\
\hline$P_{1,(n-1)}^{k}=0$, any value for $a_{2}^{k}$ and $c_{2}^{k}(\mathrm{IW})$ & {$\left[\frac{1}{\mu_{2, n}}-\frac{1}{b_{2,(n-1)}^{k}}\right]^{+}$} \\
\hline$P_{1,(n-1)}^{k} \neq 0,\left\{a_{2}^{k}<0 ; c_{2}^{k}>0\right\}(\mathrm{SIC} 1)$ & 0 \\
\hline $\begin{array}{l}P_{1,(n-1)}^{k} \neq 0,\left\{a_{2}^{k}=0 ; c_{2}^{k}=0\right\} \text { (Weak Int.) } \\
\text { or }\left\{a_{2}^{k}>0 ; c_{2}^{k}<0\right\}(\text { SIC } 3)\end{array}$ & $\min \left\{\left[\frac{1}{\mu_{2, n}}-\frac{1}{b_{2,(n-1)}^{k}}\right]^{+} ; \frac{l_{\mathrm{th}}^{k}}{\left|h_{12}^{k}\right|^{2}}\right\}$ \\
\hline$P_{1,(n-1)}^{k} \neq 0,\left\{a_{2}^{k}<0 ; c_{2}^{k}<0\right\}(\mathrm{SIC} 2)$ & $\min \left\{\frac{c_{2}^{k}}{a_{2}^{k}} ;\left[\frac{1}{\mu_{2, n}}-\frac{1}{b_{2,(n-1)}^{k}}\right]^{+} ; \frac{l_{h}^{k}}{\left|h_{12}^{k}\right|^{2}}\right\}$ \\
\hline $\begin{array}{l}P_{1,(n-1)}^{k} \neq 0,\left\{a_{2}^{k}>0 ; c_{2}^{k}>0\right\}(\text { SIC 4) } \\
\text { if } \frac{c_{2}^{k}}{a_{2}^{k}} \leqslant \frac{1 t_{\mathrm{th}}^{k}}{\left|h_{12}^{k}\right|^{2}} \\
\text { otherwise }\end{array}$ & $\min \left\{\max \left\{\left[\frac{1}{\mu_{2, n}}-\frac{1}{b_{2,(n-1)}^{k}}\right]^{+} ; \frac{c_{2}^{k}}{a_{2}^{k}}\right\} ; \frac{l_{\mathrm{th}}^{k}}{\left|h_{12}^{k}\right|^{2}}\right\}$ \\
\hline
\end{tabular}
$\left\{a_{2}^{k}>0 ; c_{2}^{k}>0\right\}$, condition (12) is equivalent to $P_{2}^{k} \geqslant$ $c_{2}^{k} / a_{2}^{k}$, so $P_{2}^{k}$ is lower-bounded by $c_{2}^{k} / a_{2}^{k}$ and upperbounded by the interference constraint and is equal to the waterfilling solution within these bounds. Of course this

Table II. Optimised values of $P_{2, n}^{k}$.

aSIC, successive interference cancellation. 
only holds if $c_{2}^{k} / a_{2}^{k} \leqslant I_{\mathrm{th}}^{k} /\left|h_{12}^{k}\right|^{2}$, otherwise there is no solution and $P_{2}^{k}$ is set to zero.

Each optimization problem on $\mathbf{P}_{1, n}$ and $\mathbf{P}_{2, n}$ is locally convex and can be easily solved using a direct waterfilling (for $\mathbf{P}_{1, n}$ ) or an adapted waterfilling (for $\mathbf{P}_{2, n}$ ). Although we can not prove that the iterative algorithm on these two variables converges to a stable state, simulations show that both primary and secondary rates converge within $1 \%$ after only two iterations. This is due to the low interference level at the primary receiver and the use of SIC at the secondary receiver when its interference level is high.

\section{RESOURCE ALLOCATION AND DECODING STRATEGY WITH TWO SECONDARY CELLS AND POSSIBLE EXTENSIONS}

\subsection{Studied scenario and assumptions}

In this section, we study a more realistic case where two secondary cells, denoted by cells 2 and 3, share the bandwidth. The system model is given by Equation (2) and detailed in Section 2.1.2

Both BS cooperate in order to maximise the data rate achieved by their mobile terminals transmission. Cooperation is performed initially, during the subcarrier allocation procedure. Subcarrier allocation is performed separately from power allocation. In the subcarrier allocation step, we assume equal power allocation. This assumption is very usual in multi-carrier resource allocation, because joint optimizations are complex and do not really improve the performances [25,26]. A multiple access strategy is used: only the user that achieves the highest estimated data rate $\tilde{R}_{i}^{k}$ in each subcarrier $k$ is allowed to transmit. The secondary terminals are assumed to transmit synchronously, and synchronously with the primary terminal. After subcarrier allocation is performed, each secondary system independently optimises its decoding strategy and power allocation, with the same method as the one presented in Section 3 when only one secondary system was active.

\subsection{Subcarrier allocation}

On each subcarrier, the data rate that can be achieved on links 2 and 3 assuming equal power allocation on all subcarriers is evaluated. This data rate is obtained using the decoding strategy, according to Table I, and taking into account the power limitation due to the interference threshold.

$$
\tilde{R}_{i}^{k}=\frac{B}{L} \log _{2}\left(1+\tilde{b}_{i}^{k} \tilde{P}_{i}^{k}\right)
$$

for $i=\{2,3\}$, where $\tilde{b}_{i}^{k}$ is given by Table I, with index 2 replaced by index $i$, and with equal power allocation on all subcarriers of the primary user. $\tilde{b}_{i}^{k}$ consequently does not depend on the iteration $n$, and the interfering power is fixed: $\tilde{P}_{1}^{k}=P_{1, \max } / L$. The power per subcarrier on each secondary link $\tilde{P}_{i}^{k}$ is given by Table II (replacing all 2 by $i=\{2,3\}$ ), where the waterfilling solution $\left[\frac{1}{\mu_{i, n}}-\frac{1}{b_{i,(n-1)}^{k}}\right]^{+}$is replaced by $P_{i, \max } / l_{i}$ for each $i=\{2,3\} . l_{i}$ is the estimated number of subcarriers allocated to cell $i$, with $l_{2}+l_{3}=L$. Finally, the set of subcarriers allocated to user $i$ is

$$
\mathcal{S}_{i}=\left\{k \in\{1, \ldots, L\} \mid i=\arg \max _{j \in\{2,3\}} \tilde{R}_{j}^{k}\right\}
$$

\subsection{Power allocation and decoding strategy}

Once subcarriers have been allocated, the algorithm proposed for the single-secondary cell case can be extended to this scenario. The power allocation algorithm for the primary user is unchanged. Then, the decoding strategy and power allocation are run for each secondary user independently. The optimization problem for each secondary user $i=\{2,3\}$ at iteration $n$ becomes

$$
\begin{aligned}
& \max _{\left\{\mathbf{P}_{i, n}\right\}} \frac{B}{L} \sum_{k \in \mathcal{S}_{i}} \log _{2}\left(1+b_{i,(n-1)}^{k} P_{i, n}^{k}\right) \\
& \text { s.t. } a_{i}^{k} P_{i}^{k} \geqslant c_{i}^{k} \forall k \in \mathcal{S}_{i} \\
& \text { s.t. }\left|h_{1 i}^{k}\right|^{2} P_{i}^{k} \leqslant I_{\mathrm{th}}^{k} \forall k \in \mathcal{S}_{i} \text { if } P_{1,(n-1)}^{k}>0 \\
& \text { s.t. } P_{i}^{k} \geqslant 0 \forall k \in \mathcal{S}_{i} \\
& \text { s.t. } \sum_{k \in \mathcal{S}_{i}} P_{i}^{k} \leqslant P_{i, \max }
\end{aligned}
$$

where $b_{i,(n-1)}^{k}, a_{i}^{k}$ and $c_{i}^{k}$ are given by Table I with index 2 replaced by index $i$. Each optimization problem is solved separately for each secondary cell. The optimised values of $P_{i, n}^{k}$ are given by Table II (again, replacing index 2 by index $i$ ) for $k \in \mathcal{S}_{i}$ and by $P_{i, n}^{k}=0$ for $k \notin \mathcal{S}_{i}$.

Similarly to the single-secondary cell case, the proposed iterative algorithm numerically converges to a stable state with $1 \%$ after two iterations in average.

\subsection{Extension to other scenarios}

\subsubsection{Uplink multi-user case.}

The two scenarios previously studied assumed only one user per cell. Extension to the multi-user case is straigthforward if all users and cells are cooperating for multiple access. Let us suppose that we still have two secondary cells indexed by 2 and 3 and that there are $U_{i}$ users on cell $i=2,3$ with $m=1, \ldots, U_{2}$ referring to the users of cell 2 and $m=U_{2}+1, \ldots, U_{2}+U_{3}$ referring to the users of cell 3 . Then cooperative subcarrier allocation is performed by selecting the following set of subcarriers for user $m \in\left\{1, \ldots, U_{2}+U_{3}\right\}$ : 
$\mathcal{S}_{m}=\left\{k \in\{1, \ldots, L\} \mid m=\arg \max _{j \in\left\{1, \ldots, U_{2}+U_{3}\right\}} \tilde{R}_{j}^{k}\right\}$

with $\tilde{R}_{j}^{k}$ the data rate evaluated with equal power allocation and given in Section 4.2. Then power allocation is performed independently for each user, by solving problem (16), where index $i$ is replaced by index $m=1, \ldots, U_{2}+U_{3}$.

\subsubsection{Downlink multi-user case.}

If we consider the downlink with one user per cell, the problem is unchanged compared with the uplink singleuser case, except that the maximum power is that of the BS. This implies that the maximum power per cell is higher and that higher data rates will be achieved.

For the downlink multi-user case, subcarrier allocation can be performed as in the uplink multi-user case, if BSs are cooperating. Then the power optimization problem per BS is written as Equation (16), with $\mathcal{S}_{i}=\{1, \ldots, L\}$, and all coefficients $b_{i,(n-1)}^{k}, a_{i}^{k}, c_{i}^{k}$ and $h_{1 i}^{k}$ are relative to the user allocated by BS $i$ in subcarrier $k . P_{i}^{k}$ is the transmit power of BS $i$ in subcarrier $k$. This problem can still be solved by the proposed algorithm.

\subsubsection{Overall interference condition.}

The optimization problem (16) has considered an interference constraint per subcarrier. This assumption may however not reflect some cognitive scenarios, where the primary system should be protected by an overall interference condition on all subcarriers. The corresponding optimization problem with two secondary cells in uplink and with one user per cell is the same as Equation (16), but the second condition is replaced by

$$
\sum_{k \in \tilde{\mathcal{S}}_{i}}\left|h_{1 i}^{k}\right|^{2} P_{i}^{k} \leqslant I_{\mathrm{th}, \max }
$$

where $\tilde{\mathcal{S}}_{i}=\left\{\mathcal{S}_{i} \mid P_{1, n-1}^{k} \neq 0\right\}$ and $I_{\text {th, max }}$ is the sum interference constraint. Solving this convex optimization problem with the Karush-Kuhn-Tucker conditions leads to the solution in Table II (with index $i$ instead of index 2) for $k \in$ $\mathcal{S}_{i}$ and by $P_{i, n}^{k}=0$ for $k \notin \mathcal{S}_{i}$, where $\left[\frac{1}{\mu_{2, n}}-\frac{1}{b_{2,(n-1)}^{k}}\right]^{+}$ is replaced by $\left[\frac{1}{\mu_{2, n}+\lambda_{2, n}\left|h_{1 i}^{k}\right|^{2}}-\frac{1}{b_{2,(n-1)}^{k}}\right]^{+}$on subcarriers $k \in \tilde{\mathcal{S}}_{i}$, and left unchanged if $k \in \mathcal{S}_{i} \backslash \tilde{\mathcal{S}}_{i}$.

As there are now two Lagrange multipliers $\mu_{2, n}$ and $\lambda_{2, n}$, the power values are more difficult to obtain, and it is necessary to use ellipsoid or interior point methods with a complexity in $\mathcal{O}\left(L^{3}\right)$ [27]. Some lowcomplexity algorithms may be used to obtain a suboptimal solution [28]. Because of lack of space, this problem cannot be solved in details in this paper and is left for further study.

\section{PERFORMANCE EVALUATION}

\subsection{Performance evaluation with one secondary cell}

We first evaluate the performances of the proposed algorithm when one secondary cell (cell 2 on Figure 1) is present. The performances of the proposed algorithm for cognitive radio are assessed using Monte Carlo simulations on the location of the primary and of the secondary mobile terminal, which both follow a uniform distribution in their cell. The complex channel gains $h_{i j}^{k}$ take into account path loss, log-normal shadowing and Rayleigh fading. We suppose here that all subcarriers are subject to independent Rayleigh fadings. The path loss model is COST 231 extension to Hata model at $800 \mathrm{MHz}$ in dense urban environment, $L_{d B}(d)=125.08+35.22 \times \log _{10}(d)$ [29], and shadowing's standard deviation is $6 \mathrm{~dB}$. Primary and secondary cells both have omnidirectional antennas, with the same radius $d_{1}=d_{2}=1 \mathrm{~km}$. The maximum power per transmitter is $21 \mathrm{dBm}$. The thermal noise has a spectral density of $-174 \mathrm{dBm} / \mathrm{Hz}$. The number of subcarriers is $L=64$, and $B=0.5 \mathrm{MHz}$. The allowed data rate decrease because of interference on the primary system is $\lambda=0.1$, which means that $90 \%$ of the interference-free rate is guaranteed. The influence of the distance between the primary and secondary BS, $d_{\text {secondary, } 2}=d_{\text {secondary }}$, will be evaluated in the simulations.

In the following, we denote our algorithm by 'AS' (adaptive strategy). It is compared with three different algorithms:

- First, the reference case when the secondary system is always forbidden to transmit (denoted as 'RP' for 'reference on primary' ).

- Second, a classical power allocation scheme in cognitive underlay/interweave system, where the secondary system can transmit on the whole bandwidth, but it considers the primary system's interference as noise in all subcarriers. This algorithm is denoted as 'FB' for 'full band'.

- Third, an algorithm where the secondary system can only transmit in the subcarriers with weak interference $\left(\left|h_{21}^{k}\right|^{2} \leqslant\left|h_{22}^{k}\right|^{2}\right.$ ), but it still sees the primary system's interference as noise in these subcarriers. This algorithm is denoted ' $\mathrm{PB}$ ' for 'partial band'.

The rates represented in the section are normalised by bandwidth B.

\subsubsection{Comparison with exhaustive search.}

First, the proposed strategy is compared with an exhaustive search, which chooses the best solution among treating interference as noise at the secondary receiver, performing SIC at the secondary receiver (under condition (12)) or forbidding the secondary link to be active. In order to keep a 
reasonable complexity, we suppose here equal power allocation on all $L$ subcarriers and only optimise the decoding strategy. Indeed, if power allocation is allowed, and even in the simple case when interference is treated as noise, the problem of maximising the sum rate considering both primary and secondary cells jointly is not convex and cannot be directly solved. Adding the decoding strategy leads to a nonconvex problem with mixed noninteger (the power) and integer (the strategies) variables. This problem becomes nontractable.

With equal power allocation, the power per carrier of the secondary user is limited to $P_{2, \max } / L$ for the secondary user but may be lower because of the interference constraint. With the proposed algorithm, the secondary power values $\hat{P}_{2}^{k}$ are given by Table II, in which the waterfilling solution $\left[\frac{1}{\mu_{2, n}}-\frac{1}{b_{2,(n-1)}^{k}}\right]^{+}$is always replaced by $P_{2, \max } / L$, and the interweave case does not take place because power is equally shared on the subcarriers of the primary transmitter. The secondary rate is then equal to $\hat{R}_{2}=\frac{B}{L} \sum_{k=1}^{L} \log _{2}\left(1+\hat{b}_{2}^{k} \hat{P}_{2}^{k}\right)$ where $\hat{b}_{2}^{k}$ is given by Table I, replacing all $P_{1,(n-1)}^{k}$ values by $P_{1, \max } / L$.

The exhaustive search chooses the strategy that maximises the sum rate. Because power is equally shared on all subcarriers, it can be computed independently per subcarrier.

The secondary rate obtained with AS is degraded compared with the exhaustive search allocation. The difference in percentage is provided in Table III, with AS but also with FB and PB. We can see, however, that the proposed method AS limits this rate loss to $6.16 \%$ and that this loss decreases when the distance between the primary and secondary cells increases. The rate losses are far higher with PB and FB. We can also notice that the sum rate loss compared with the exhaustive search solution is almost negligible (and thus not shown here) with our proposed method.

Table III. Secondary rate loss (\%) compared with the exhaustive search strategy.

\begin{tabular}{lccc}
\hline$d_{\text {secondary }}(\mathrm{km})$ & AS & FB & PB \\
\hline 0 & 6.16 & 14.06 & 24.69 \\
0.1 & 5.54 & 16.41 & 26.21 \\
0.2 & 5.34 & 17.18 & 26.55 \\
0.3 & 4.87 & 16.00 & 24.77 \\
0.4 & 4.21 & 15.37 & 22.88 \\
0.5 & 3.66 & 13.91 & 20.44 \\
0.6 & 3.06 & 13.17 & 18.66 \\
0.7 & 2.80 & 11.01 & 15.91 \\
0.8 & 2.47 & 9.04 & 13.27 \\
0.9 & 2.20 & 6.63 & 10.30 \\
1 & 1.97 & 4.8 & 8.09 \\
\hline
\end{tabular}

${ }^{\mathrm{b}} \mathrm{AS}$, adaptive strategy; FB, full band; $\mathrm{PB}$, partial band.

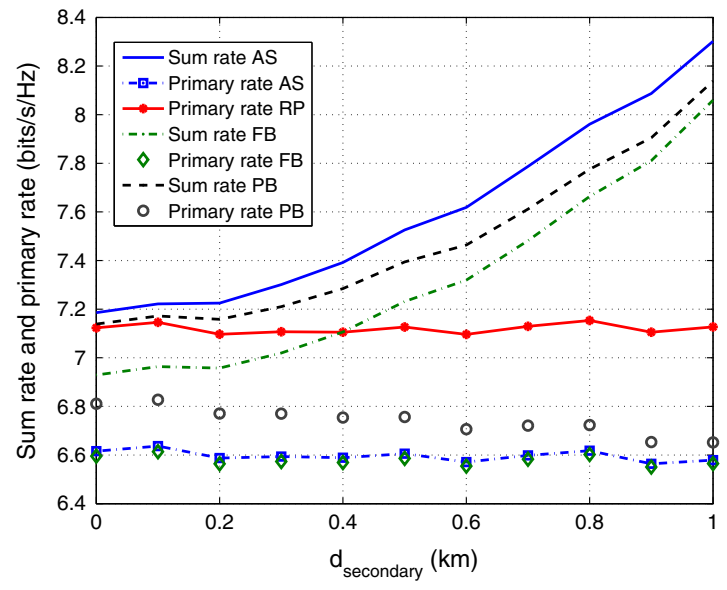

Figure 3. Sum rate and primary rate, one secondary cell.

\subsubsection{Rates comparison.}

The sum rate and the primary rate are shown on Figure 3. The highest sum rate is achieved with the proposed method AS. When the secondary system is active, the sum rate increases with $d_{\text {secondary. The sum rate gain }}$ obtained with AS compared with RP is between 0.9 and $16.5 \%$, and it increases with $d_{\text {secondary }}$. The data rate gain is $3.0-4.1 \%$ and $0.7-2.4 \%$ compared with $\mathrm{FB}$ and $\mathrm{PB}$, respectively. It increases when $d_{\text {secondary }}$ is between 0 and $0.7 \mathrm{~km}$ and decreases afterwards. One may expect that AS, FB and PB will behave almost similarly at very high distances, when treating interference as noise would be opti$\mathrm{mal}$ in all the cases, except for the interweave ones.

FB and PB algorithms provide higher primary rates than AS but lower sum rates. The decrease in primary rate with AS is however not an issue, because a degradation of up to $10 \%$ can be tolerated, according to the interference limitation threshold. Figure 3 shows that the primary rate decrease is in fact lower, around $7.3 \%$, and it is almost independent of the distance $d_{\text {secondary }}$.

Figure 4 shows that AS provides a strong improvement of the secondary rates, especially when $d_{\text {secondary }}$ is low. The secondary rate increase with AS is at least $15.2 \%$ and $15.9 \%$, compared with FB and PB, respectively. Treating interference as noise on all subcarriers (with FB) or on a subset of them (with $\mathrm{PB}$ ) does not really change the secondary user's rate. This proves that FB is not efficient on the subcarriers where the secondary receiver is under strong interference, because treating interference as noise then leads to almost negligible data rates.

\subsubsection{Distribution of cases and of data rates.}

In order to better understand the impact of the decoding strategy on the rate results, we now evaluate the distribution of cases, depending on $d_{\text {secondary. Figure } 5 \text { shows }}$ that SIC cases mainly happen when $d_{\text {secondary }}$ is low or medium, whereas a large majority of the cases corresponds to weak interference when the secondary BS is far from the 


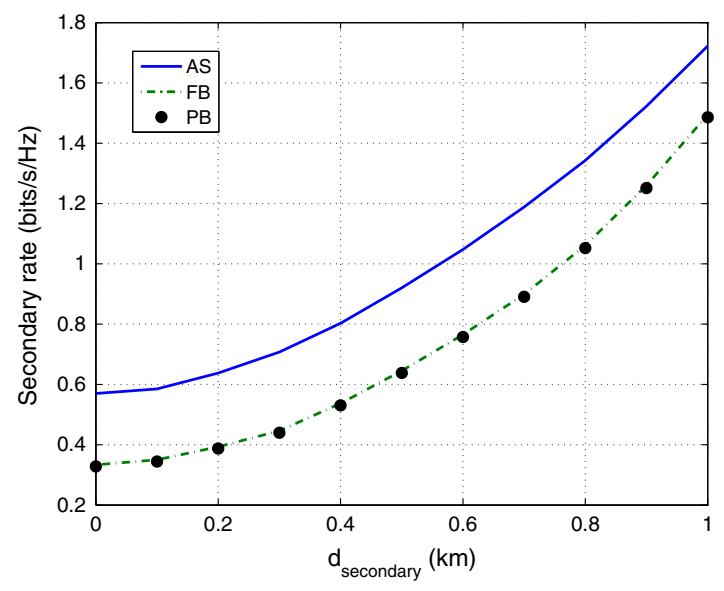

Figure 4. Secondary rate, one secondary cell.

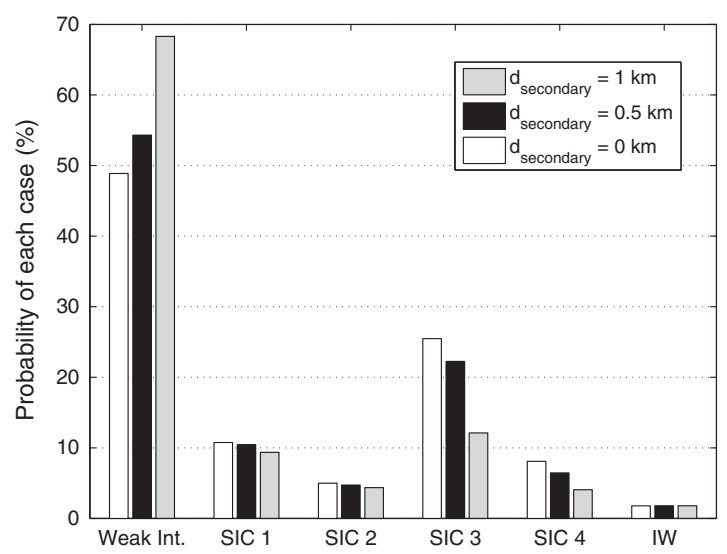

Figure 5. Probability of interference cases in cognitive scenario, one secondary cell.

primary BS. Interweave cases are very rare, less than $2 \%$. The average primary rate per subcarrier is represented on Figure 6 . The primary rate decreases with $d_{\text {secondary }}$ in SIC cases but slightly increases with $d_{\text {secondary }}$ when interference is weak. It is interesting to notice that the primary rate is not higher in weak interference cases than in SIC cases, except of course when the secondary rate is inactive (SIC 1 and some subcases of SIC 4). These results show that the proposed strategy efficiently adapts to the interference cases.

\subsection{Performance evaluation with two secondary cells}

In this section, we evaluate the performances of the proposed algorithm with two cooperating secondary cells. The simulation hypotheses are the same as in the previous section, but an additional secondary cell is present (see Figure 1 and the assumptions in Section 2.1.

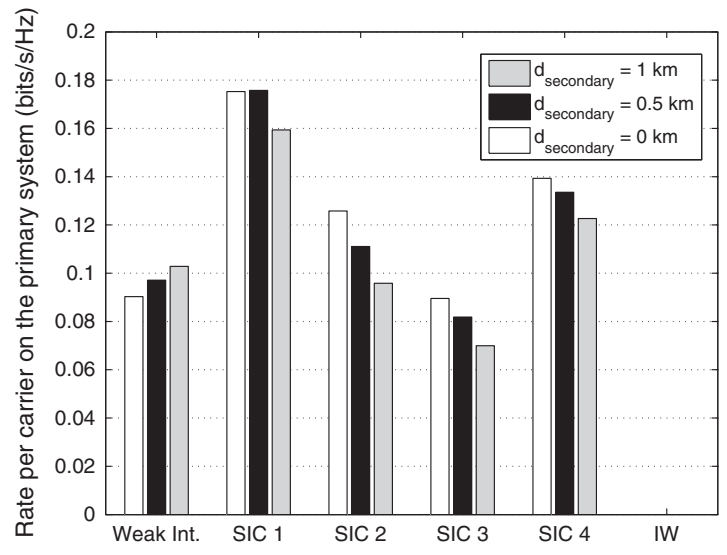

Figure 6. Primary rate per subcarrier, depending on the cases, one secondary cell.

In order to make a fair comparison between the proposed algorithm (AS) and the other ones, a specific subcarrier allocation procedure among the secondary cells is used for algorithms FB and PB. Each subcarrier is allocated to the secondary user that achieves the highest estimated data rate, assuming equal power allocation on all subcarriers at the primary and secondary users. The estimated data rate is computed supposing that the secondary system considers interference as noise: $\tilde{R}_{i}^{k}=\frac{B}{L} \log _{2}\left(1+\left|h_{i i}^{k}\right|^{2} \tilde{P}_{i}^{k} /\left(n_{0}+\left|h_{i 1}^{k}\right|^{2} \tilde{P}_{1}^{k}\right)\right)$ for $i \in\{2,3\})$. The values of $\tilde{P}_{i}^{k}$ for $i \in\{1,2,3\}$ are the same as for AS algorithm (Section 4.2).

\subsubsection{Performances when both secondary base stations are at the same distance from the primary base station.}

In a first scenario, we suppose that both secondary BSs are at the same distance from the primary BS, $d_{\text {secondary }, i}=d_{\text {secondary }}$ for $i \in\{2,3\}$. The influence of this distance is then evaluated. As both secondary BSs should have statistically the same behaviour (which is verified by simulations, in the subcarrier allocation phase, the estimated number of subcarriers per BS is set to $l_{i}=L / 2$ for $i \in\{2,3\}$.

The highest sum rate, represented on Figure 7, is still obtained with the proposed method. The improvement is higher than when only one secondary cell was active, compared with RP (6.8-30.5\%), and it increases with the distance. The data rate gain is $0-1.6 \%$ with FB and $3.3-4.8 \%$ with $\mathrm{PB}$ and behaves similarly as in the single-secondary cell case. Nevertheless, contrary to the single-secondary cell case, FB is here more efficient than PB. This is due to the multi-user diversity, which increases the probability of assigning subcarriers in favourable weak interference conditions to each secondary user.

Figure 8 aggregates the data rates on both secondary cells. The average rate per secondary cell is statistically the 


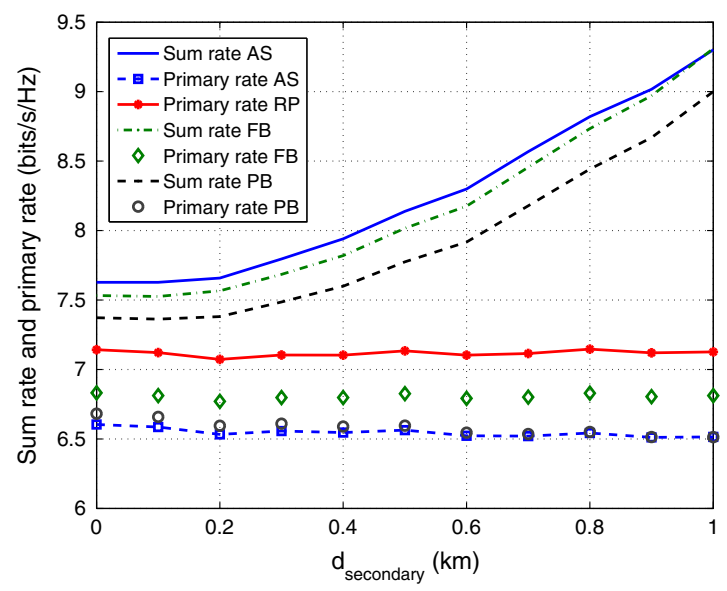

Figure 7. Sum rate and primary rate, two secondary cells at the same distance.

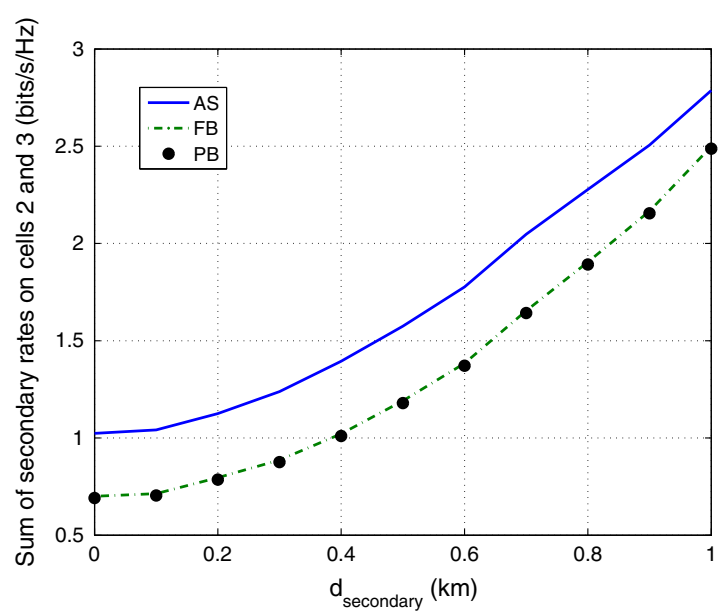

Figure 8. Secondary rates, two secondary cells at the same distance.

same on each cell, because they are at the same distance from the primary BS. The secondary data rate increase compared with FB and PB is very high when the distance is low and tends to decrease when $d_{\text {secondary }}$ increases. However, it always remains higher than $11.5 \%$ and $12.0 \%$, respectively. Compared with the case with one secondary cell, the data rate per secondary user is decreased of 10.2 to $19.0 \%$, even though each user only gets half of the subcarriers. Consequently, the decoding strategy becomes more efficient thanks to multi-user diversity. One interesting extension of these results would be to study if these conclusions still apply to more than two secondary systems.

Finally, the primary rate (shown on Figure 7) decreases of around $8 \%$ compared with RP, which is a bit higher than with one secondary cell but still lower than the degradation of $10 \%$ tolerated by the system.

Figure 9 represents the distribution of cases. It shows that subcarrier allocation slightly favours weak interference cases.

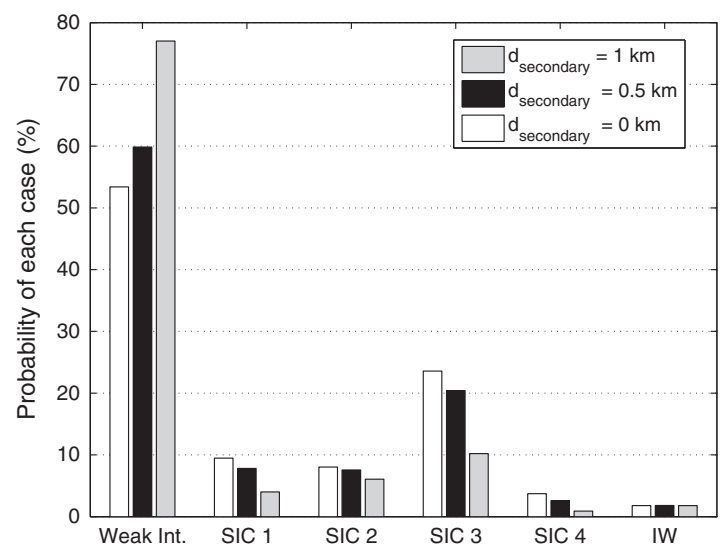

Figure 9. Probability of interference cases in cognitive scenario, two secondary cells at the same distance.

Table IV. Percentage of subcarriers allocated to cell 3 depending on the algorithm.

\begin{tabular}{lcc}
\hline$d_{\text {secondary }, 3}(\mathrm{~km})$ & AS & FB and PB \\
\hline 0 & 43.4 & 45.2 \\
0.1 & 43.5 & 45.3 \\
0.2 & 44.0 & 46.0 \\
0.3 & 44.6 & 46.5 \\
0.4 & 46.3 & 48.6 \\
0.5 & 50.0 & 50.0 \\
0.6 & 50.8 & 52.1 \\
0.7 & 52.2 & 55.0 \\
0.8 & 54.1 & 58.0 \\
0.9 & 56.6 & 62.0 \\
1 & 59.2 & 65.8 \\
\hline
\end{tabular}

${ }^{\mathrm{C}} \mathrm{AS}$, adaptive strategy; FB, full band; $\mathrm{PB}$, partial band.

\subsubsection{Influence of the secondary base station distances.}

In a second scenario, the influence of the relative distance between the secondary BS is studied. Here, we suppose that $d_{\text {secondary, } 2}=0.5 \mathrm{~km}$, and we modify the value of $d_{\text {secondary, } 3}$ from 0 to $1 \mathrm{~km}$. To simplify, the estimated number of subcarriers per BS is set to $l_{i}=L / 2$ for $i \in\{2,3\}$.

Table IV contains the percentage of subcarriers allocated to cell 3 with the two different subcarrier allocation algorithms. PB and FB favour cell 3 when $d_{\text {secondary, } 3}$ increases, that is, when its BS receives less interference from the primary user, leading to higher estimated data rates because interference is then always treated as noise. With our proposed algorithm, the increase is less marked, because a user close to the primary BS may get the subcarrier, thanks to SIC. Finally, we can notice that the variance is not high around the mean of $50 \%$, which allows us to set $l_{i}=L / 2$ for $i \in\{2,3\}$ with reasonable estimation error at the subcarrier allocation step.

Figure 10 shows the influence of $d_{\text {secondary, } 3}$ on the sum and primary rates. The sum rate increase compared with 


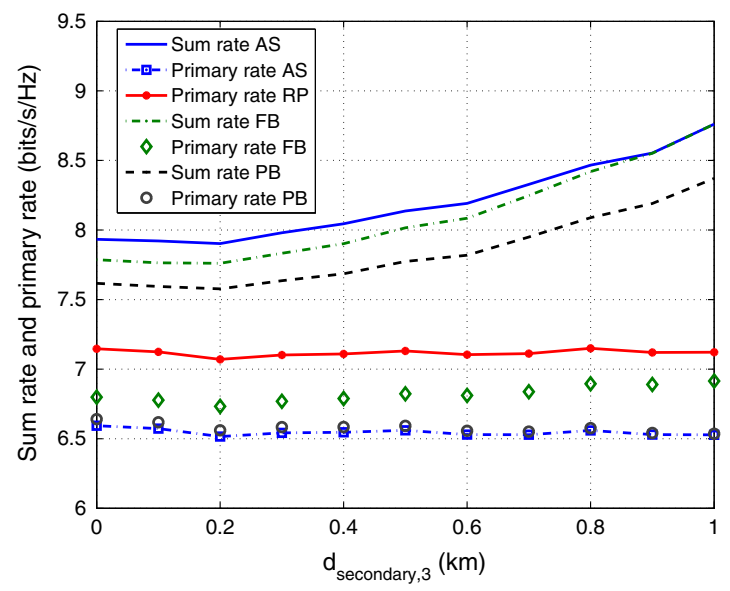

Figure 10. Sum rate and primary rate, cell 3 at varying distance.

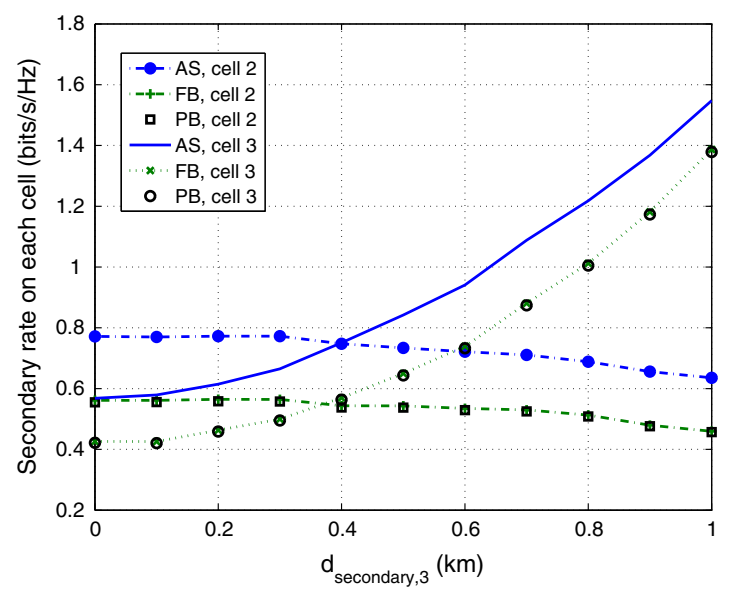

Figure 11. Secondary rates, cell 3 at varying distance.

RP is between $11.0 \%$ and $22.3 \%$. The secondary rates are represented on Figure 11. AS provides the highest rates on cells 2 and 3. The best improvements are achieved at low $d_{\text {secondary, } 3}$, which corresponds to higher interference situations, when adapting the decoding strategy is more useful. Still, even when the distance is high and weak interference cases prevail, our proposed method allows to increase the rate on cell 3 of 33.3-11.6\% and 34.7-12.2\% compared with $\mathrm{FB}$ and $\mathrm{PB}$, respectively. It is consequently robust to the distance variations.

\section{CONCLUSIONS}

In this paper, an adaptive resource allocation and decoding strategy in a cognitive radio system has been proposed. This heuristic is based on the G-IFC sum-capacity achieving strategy and is here adjusted to the specific case of underlay and interweave cognitive radio with the objective to maximise both primary and secondary rates. The proposed algorithm allows the secondary systems to reach reasonable data rates, at the expense of a low rate decrease on the primary system. It is more effective than classical algorithms, where interference is treated as noise at the secondary receivers. Cooperation between the secondary cells regarding subcarrier allocation allows to achieve low degradation of each secondary user's data rate compared to the single-secondary cell case, even though each user only gets half of the subcarriers. Finally, the proposed algorithm adapts to the locations of the secondary cells, by selecting the best decoding strategy adequately, and this still holds if the secondary cells have different locations. Future work will consist in determining more general algorithms with any number of secondary systems, and evaluating their cost in terms of required CSI.

\section{ACKNOWLEDGEMENTS}

This work is partially supported by the European project EMPhAtiC (ICT- 318362).

\section{REFERENCES}

1. Mitola J. Cognitive radio for flexible mobile multimedia communications, In Proc. IEEE Int. Work. Mobile Multi. Commun., San Diego, CA, 1998.

2. Zhao Q, Sandler BM. A survey of dynamic spectrum access. IEEE Signal Processing Magazine 2007; 24(3): 79-89.

3. Goldsmith A, Jafar S, Maric I, Srinivasa S. Breaking spectrum gridlock with cognitive radios: an information theoretic perspective. Proceedings of IEEE 2009; 97(5): 894-914.

4. Haykin S. Cognitive radio: brain-empowered wireless communications. IEEE Journal on Selected Areas in Communications 2005; 23(2): 201-220.

5. Jiang J, Xin Y. On the achievable rate regions for interference channels with degraded messages sets. IEEE Transactions on Information Theory 2008; 54(10): 4707-4712.

6. Maric I, Goldsmith A, Kramer G, Shamai(Shitz) S. On the capacity of interference channels with one cooperating transmitter. European Transactions on Telecommunications (ETT) 2008; 19(4): 405-420.

7. Devroye N, Mitran P, Tarokh V. Achievable rates in cognitive radio channels. IEEE Transactions on Information Theory 2006; 52(5): 1813-1827.

8. Gastpar M. On capacity under receive and spatial spectrum-sharing constraints. IEEE Transactions on Information Theory 2007; 53(1): 471-487.

9. Ghasemi A, Sousa ES. Fundamental limits of spectrumsharing in fading environments. IEEE Transactions on Information Theory 2007; 6(2): 649-658.

10. Musavian L, Aissa S. Capacity and power allocation for spectrum-sharing communications in fading channels. 
IEEE Transactions on Wireless Communications 2009; 8(1): 148-156.

11. Han T, Kobayashi K. A new achievable rate region for the interference channel. IEEE Transactions on Information Theory 1981; 27: 49-60.

12. Shang X, Kramer G, Chen B. A new outer bound and the noisy-interference sum-rate capacity for the Gaussian interference channel. IEEE Transactions on Information Theory 2009; 55: 689-699.

13. Motahari A, Khandani AK. Capacity bounds for the Gaussian interference channel. IEEE Transactions on Information Theory 2009; 55: 620-643.

14. Pischella M, Le-Ruyet D. Power allocation for the multicarrier two-user Gaussian interference channel, In Proc. IEEE SPAWC, Cesme, Turkey, 2012.

15. Tuninetti D. Gaussian fading interference channels: power control, In Proc. Asilomar, Pacific Grove, USA, 2008.

16. Shaat M, Bader F. Efficient resource allocation algorithm for uplink in multicarrier-based cognitive radio networks with fairness consideration. IET Communications Journal 2011; 5(16): 2328-2338.

17. Digham F. Joint power and channel allocation for cognitive radios, In Proc. IEEE Wireless Commun. and Networking Conf., Las Vegas, NV, USA, 2008.

18. Zayen B, Haddad M, Hayar A, Oien GE. Binary power allocation for cognitive radio networks with centralized and distributed user selection strategies. Physical Communication Journal, Elsevier 2008; 1(3): 183-193.

19. Zhang H, Le-Ruyet D, Roviras D, Medjahi Y, Sun H. Spectral efficiency comparison of OFDM/FBMC for uplink cognitive radio networks. EURASIP Journal on Advances in Signal Processing 2010; 10. id: 621808.
20. Etkin R, Tse D, Wang H. Gaussian interference channel capacity to within one bit. IEEE Transactions on Information Theory 2008; 54: 5534-5562.

21. Bresler G, Tse D. The two-user Gaussian interference channel: a deterministic view. European Transactions on Telecommunications 2008; 19(4): 333-354.

22. Sato H. The capacity of the Gaussian interference channel under strong interference. IEEE Transactions on Information Theory 1981; 27: 786-788.

23. Zhang H, Le-Ruyet D, Roviras D, Sun H. Noncooperative multi-cell resource allocation of FBMC based cognitive radio systems. IEEE Transactions on Vehicular Technology 2012; 61(2): 799-811.

24. Boyd S, Vanderbergue L. Convex Optimization. Cambridge University Press: New York, USA, 2004.

25. Rhee W, Cioffi JM. Increase in capacity of multiuser OFDM system using dynamic subchannel allocation, In Proc. Veh. Tech. Conf., Tokyo, Japan, 2000.

26. Shen Z, Andrews J, Evans B. Adaptive resource allocation in multiuser OFDM systems with proportional rate constraints. IEEE Transactions on Wireless Communications 2005; 4(6): 2726-2737.

27. Zhang Y, Leung C. An efficient power-loading scheme for OFDM-based cognitive radio systems. IEEE Transactions on Vehicular Technology 2010; 59(4): 1858-1864.

28. Shaat M, Bader F. Computationally efficient power allocation algorithm inmulticarrier-based cognitive radio networks: OFDMand FBMC systems. EURASIP Journal on Advances in Signal Processing 2010; 2010: 1-13. Article ID 528378.

29. Goldsmith A. Wireless Communications. Cambridge University Press: New York, USA, 2005. 